\title{
Scaling properties for a classical particle in a time-dependent potential well
}

\author{
Edson D. Leonel ${ }^{\text {a) }}$ and P. V. E. McClintock \\ Department of Physics, Lancaster University, Lancaster, LA1 4YB, United Kingdom
}

(Received 14 January 2005; accepted 5 May 2005; published online 5 July 2005)

\begin{abstract}
Some scaling properties for a classical particle interacting with a time-dependent square-well potential are studied. The corresponding dynamics is obtained by use of a two-dimensional nonlinear area-preserving map. We describe dynamics within the chaotic sea by use of a scaling function for the variance of the average energy, thereby demonstrating that the critical exponents are connected by an analytic relationship. (c) 2005 American Institute of Physics. [DOI: 10.1063/1.1941067]
\end{abstract}

Scaling arguments are used to characterize the chaotic sea in the low energy domain for the problem of a classical particle confined to an infinitely deep potential box containing an oscillating square well. The system is described by three relevant control parameters via the formalism of an area-preserving map. We thus concentrate on analyzing the variance of the average energy, which we refer to as the roughness. We do so as a function of two relevant control parameters, namely: (i) the oscillating frequency; and (ii) the depth of the oscillating potential. As the map is iterated, the roughness grows to start with. For a sufficient number of iterations, however, the rate of growth decreases and a regime of saturation is entered. The changeover from growth to saturation is characterized by a crossover iteration number. Both the roughness saturation value and the crossover iteration number can be described in terms of critical exponents, and we show that the exponents differ depending on which control parameter is considered. The scaling arguments are proved by a convincing collapse of different roughness curves into a single universal plot.

\section{INTRODUCTION}

Time-dependent Hamiltonian systems have received much attention in recent years, ${ }^{1,2}$ leading to significant advances toward a qualitative and quantitative understanding of their behavior over long times. In particular, the characterization of time evolution for an ensemble of slightly different initial conditions allows one to observe the phenomenon of sensitivity to initial conditions, sometimes known as unpredictability. ${ }^{3}$ If the time evolution of the system becomes radically different as the result of slightly changed initial condition, it is an indication that the system has chaotic components. In many cases, such problems may be described in terms of area-preserving maps. The phase space may then include invariant spanning curves and KAM islands surrounded by a chaotic sea that may be characterized via Lyapunov exponents. ${ }^{4}$ Behavior of this kind (with a mixed phase space structure) is indeed generic for nondegenerate Hamiltonian systems and has been observed for problems with time-dependent potentials, ${ }^{5,6}$ mesoscopic

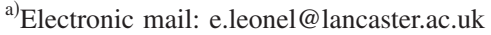

systems, ${ }^{7,8}$ waveguides, ${ }^{9}$ and for a wide class of billiards with a variety of boundaries. ${ }^{10-13}$ For the billiards problems, it is assumed that the particle moves freely inside a fixed boundary with which it collides elastically. In the formalism of Hamiltonian systems, the boundary is assumed to be a barrier of infinite height. If the boundary were timedependent, however, the scenario observed could become very different. In special cases, the particle experiences the phenomenon of Fermi acceleration and exhibits unlimited energy growth. See Ref. 14 for specific examples.

Particular effort has been devoted to understanding the problem of a particle interacting with potentials of different kinds. The special interest in studying these systems arises because they are accessible to a variety of formalisms and approaches. Moreover, not only the classical but also the corresponding quantum versions of each system can be treated. Thus, both theoretical and experimental results have been obtained for a quantum particle interacting with a potential-like barrier. These include the frequency dependence of the tunneling time, ${ }^{15}$ the transmission probability spectrum in a driven triple diode in the presence of a periodic external field, ${ }^{16}$ photon-assisted tunneling through a $\mathrm{GaAs} / \mathrm{Al}_{x} \mathrm{Ga}_{1-x} \mathrm{As}$ quantum dot induced by an external microwave field, ${ }^{17}$ sequential tunneling in a superlattice induced by an intense electric field, ${ }^{18}$ electron transmission resonance above a quantum well due to dissipation, ${ }^{19}$ and the probability of dissipative tunneling in Josephson junction circuits. ${ }^{20}$ For the classical case, in particular for systems in which a classical particle interacts with a time-dependent potential, different formalisms were developed, including treatments of the problem of a time-modulated barrier,21,22 and of a classical particle in a time-dependent oscillating well. ${ }^{23,24}$ Consideration of the dynamics of these problems in the presence of noise has recently led to exact descriptions of diffusion within static single and double-square well potentials, ${ }^{25,26}$ the introduction of an external field for twolevel systems in a classical potential, ${ }^{27}$ a general solution of the problem of activated escape in periodically driven systems, ${ }^{28}$ analytic solutions for the problem of a piecewise bistable potential in the limit of weak external perturbation, ${ }^{29}$ calculation of the escape flux from a multiwell metastable potential at times preceding the formation of quasiequilibrium, ${ }^{30}$ activation over a randomly fluctuating 


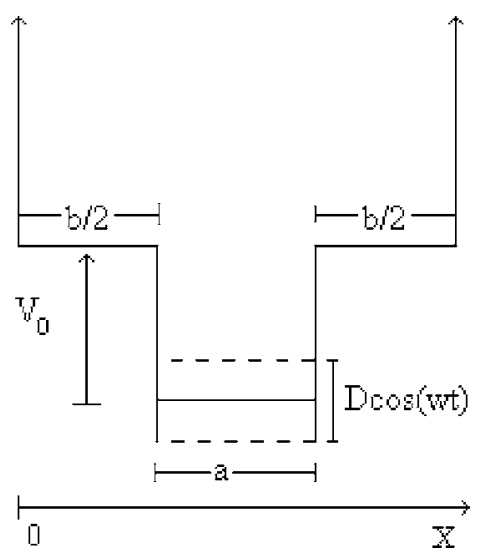

FIG. 1. Sketch of the potential $V(x, t)$ under consideration.

barrier, ${ }^{31,32}$ and diffusion across a randomly fluctuating barrier. $^{33}$

In this paper we revisit the problem of a classical particle interacting with an infinitely deep potential well containing a periodically oscillating square well, with the aim of describing some of its scaling properties. We will concentrate on the chaotic low energy region, describing the variance of the average energy by use of a scaling function. The particular formalism we use was motivated by results obtained from surface science, ${ }^{34}$ and it is one that has already proved valuable in the characterization of chaotic systems. ${ }^{35}$ It could also be useful in application to billiards problems. Using the roughening concept, we obtain a scaling function as well as critical exponents for chaotic low energy time series. The scaling function is confirmed by a convincing collapse of roughness data for a large range of different control parameters onto a single universal curve, as we shall see.

The paper is organized as follows. In Sec. II, we describe how the map is constructed. The numerical results and scaling properties are discussed in Sec. III while Sec. IV summarizes our results and presents concluding remarks.

\section{THE MODEL AND MAP DERIVATION}

We consider a particle moving inside an infinite potential box that contains a periodically oscillating square well. The system is described by the Hamiltonian $H(x, p, t)=p^{2} / 2 m$ $+V(x, t)$, where $V(x, t)$ is the potential within which the particle remains confined. It is written as

$$
V(x, t) \quad\left\{\begin{array}{ccc}
\infty & \text { if } x \leqslant 0 & \text { or } x \geqslant a+b \\
V_{0} & \text { if } 0<x<\frac{b}{2} & \text { or }\left(a+\frac{b}{2}\right)<x<(a+b) \\
D & \cos (w t) & \text { if } \frac{b}{2}<x<\left(a+\frac{b}{2}\right),
\end{array}\right.
$$

where $V_{0}, a, b, D$, and $w$ are constants. In what follows, allusions to "the well" refer to the central oscillating part of the potential $V(x, t)$, which is sketched in Fig. 1. Note that this particular geometry for the potential $V(x, t)$ makes it equivalent to the problem of an infinite chain of oscillating square wells, ${ }^{24}$ and similar to the problem of a single oscil- lating square well with periodic boundary conditions. This kind of potential can be related directly to mesoscopic systems ${ }^{16}$ with time-dependent potentials ${ }^{16,19}$ with the square well representing, e.g., the conduction band for a $\mathrm{GaAs} / \mathrm{Al}_{x} \mathrm{Ga}_{1-x} \mathrm{As}$ heterostructure with the time-dependent potential representing the electron-phonon interaction. ${ }^{36}$ Furthermore, as indicated earlier, the formalism used to derive the scaling relation for the chaotic low energy region in this problem is directly applicable to billiards problems. The same formalism was recently used for investigation of the chaotic sea in the Fermi-Ulam accelerator model. ${ }^{35}$

We now construct the map describing the dynamics of this system. We follow basically the same general procedures $^{22}$ recently applied ${ }^{5}$ to investigate classical dynamics within a time-modulated barrier. See also Ref. 23 for the problem of an oscillating single well, and Ref. 6 for the problem of an oscillating double square well. Note that the present system only differs from that discussed in Ref. 5 by virtue of the fact that here the central part of the potential is a well, whereas in Ref. 5 it was a barrier. However, this apparently minor difference in the potential may be expected to produce profound differences in the dynamics of the particle. In Ref. 5 the particle needed to surmount a central (oscillating) barrier in the potential, and thus spent significant time with its motion effectively restricted to either one or other side of the barrier; in the present case, there is no barrier, but a narrower and deeper (oscillating) central portion of the potential where the particle may sometimes be confined. Despite the apparent similarity of the two potentials, therefore, there is no a priori reason to expect any particular similarity in the dynamics. Nonetheless, as we will demonstrate in the following there are a number of striking similarities, and we will argue that they point to universality in the behavior of these kinds of systems. We will use critical exponents obtained for the scaling function describing the roughness for these two systems, and results for the traversal time and successive reflection numbers and times, to support the universality hypothesis.

We will consider as dynamical variables both the total energy of the particle $E$ and the time $t$ measured from when the particle enters the well. Suppose that, at a time $t=t_{n}$, the particle is at the entrance of the well with total energy $E$ $=E_{n}$. The formalism described here is applicable to entrance from either side of the well. When the particle enters the well, it suffers an abrupt change in its kinetic energy, which can be written as $K_{n}^{\prime}=E_{n}-D \cos \left(w t_{n}\right)$. Once it is inside the well, the particle experiences no forces, and it moves freely with a constant velocity of modulus $\left|v_{n}^{\prime}\right|=\sqrt{2 K_{n}^{\prime} / m}$. After traveling a distance $a$ in a time $t_{n}^{\prime}=a /\left|v_{n}^{\prime}\right|$, the particle reaches the other side of the well. The total energy of the particle is then $E_{n}^{\prime}=K_{n}^{\prime}+D \cos \left[w\left(t_{n}+t_{n}^{\prime}\right)\right]$. At this point, two different things may occur: (a) if the particle has insufficient energy to escape from the well, it will be reflected back with same kinetic energy. In this case, $E_{n}^{\prime}<V_{0}$. (b) The particle escapes from the well, i.e., $E_{n}^{\prime}>V_{0}$. Let us first discuss case (a). If the particle is reflected it will eventually reach the other side of the well. At this time, its total energy is $E_{n}^{\prime}=K_{n}^{\prime}$ $+D \cos \left[w\left(t_{n}+2 t_{n}^{\prime}\right)\right]$. But if $E_{n}^{\prime}<V_{0}$, the particle is reflected 
back again, and so on until the following condition is satisfied:

$$
E_{n}^{\prime}=K_{n}^{\prime}+D \cos \left[w\left(t_{n}+i t_{n}^{\prime}\right)\right]>V_{0} .
$$

Here, $i$ is the smaller integer for which Eq. (1) is true. Exiting the well, case (b) then applies. The particle again suffers an abrupt change in its kinetic energy so that the new relation is $K_{n}^{\prime \prime}=E_{n}^{\prime}-V_{0}$. In this case, the corresponding velocity is given by $\left|v_{n}^{\prime \prime}\right|=\sqrt{2 K_{n}^{\prime \prime} / m}$. The particle thus travels a distance $b / 2$ until it suffers an elastic collision with the infinite potential wall, from which it is reflected back with same kinetic energy $K_{n}^{\prime \prime}$. As it returns, it travels the distance $b / 2$ until it again enters the well. The time spent in this part of its trajectory is $t_{n}^{\prime \prime}=b /\left|v_{n}^{\prime \prime}\right|$. The map is therefore given by

$$
T:\left\{\begin{array}{l}
E_{n+1}=E_{n}+D\left[\cos \left(w\left(t_{n}+i t_{n}^{\prime}\right)\right)-\cos \left(w t_{n}\right)\right] \\
t_{n+1}=t_{n}+i t_{n}^{\prime}+t_{n}^{\prime \prime},
\end{array}\right.
$$

where $i$ is obtained as the smallest integer for which Eq. (1) is true (see above).

This form of the map involves an excessive number of control parameters. There are five in total, namely $a, b, D$, $V_{0}$, and $w$. So it is advantageous to transform to dimensionless and more convenient variables. We will define $e_{n}$ $=E_{n} / V_{0}, \delta=D / V_{0}$, and $r=b / a$. In practice it is convenient to measure time in terms of the period of oscillation of the moving well, so $\phi_{n}=w t_{n}$. We can also define a parameter $N_{c}$ given by

$$
N_{c}=\frac{w a}{2 \pi} \sqrt{\frac{m}{2 V_{0}}} .
$$

The parameter $N_{c}$ deserves a more detailed discussion. The term $a / \sqrt{2} V_{0} / m=\tau_{c}$ can be interpreted as being the time a particle spends in traveling a distance $a$ with kinetic energy $K=V_{0}$. Related to this time, we can define a characteristic frequency $\omega_{c}=2 \pi / \tau_{c}$ and then rewrite Eq. (2) as $N_{c}=w / \omega_{c}$. These considerations tell us that the parameter $N_{c}$ represents the number of oscillations that the square well completes in a time $t=\tau_{c}$. With these parameters, the map becomes

$$
T:\left\{\begin{array}{l}
e_{n+1}=e_{n}+\delta\left[\cos \left(\phi_{n}+i \Delta \phi_{a}\right)-\cos \left(\phi_{n}\right)\right] \\
\phi_{n+1}=\phi_{n}+i \Delta \phi_{a}+\Delta \phi_{b} \bmod 2 \pi,
\end{array}\right.
$$

where the auxiliary variables are given by

$$
\begin{aligned}
\Delta \phi_{a} & =\frac{2 \pi N_{c}}{\sqrt{e_{n}-\delta \cos \left(\phi_{n}\right)}}, \\
\Delta \phi_{b} & =\frac{2 \pi N_{c} r}{\sqrt{e_{n}+\delta\left[\cos \left(\phi_{n}+i \Delta \phi_{a}\right)-\cos \left(\phi_{n}\right)\right]-1}},
\end{aligned}
$$

and $i$ is the smallest integer number that makes the following equation true:

$$
e_{n}+\delta\left[\cos \left(\phi_{n}+i \Delta \phi_{a}\right)-\cos \left(\phi_{n}\right)\right]>1 .
$$

The map $T$ [see Eq. (3)] is area preserving because Det $J$ $=1$ where $J$ is the Jacobian matrix.
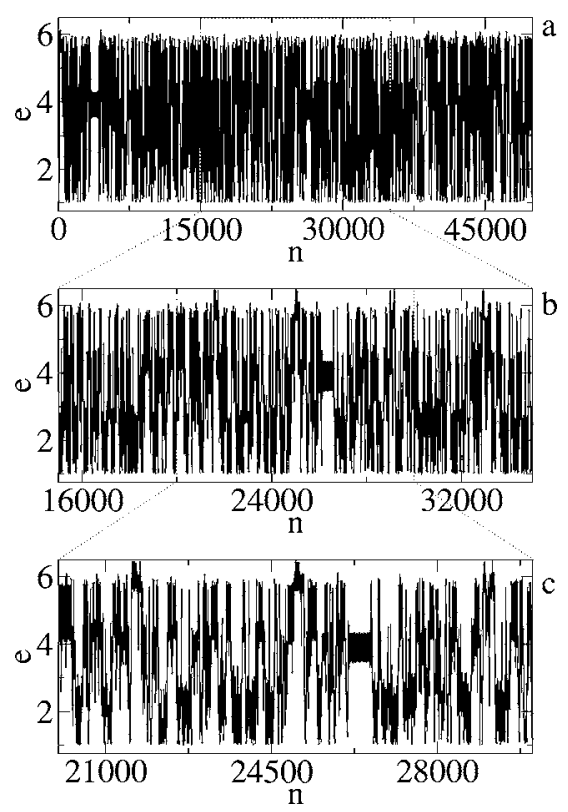

FIG. 2. Time evolution of the energy $e$ from one initial condition in the chaotic sea with the control parameters $\delta=0.5, r=1$, and $N_{c}=33.18$. Note the widely differing abscissa scale expansions and that $e(n)$ appears scale-independent.

The phase space for this problem is shown to have a hierarchy of behaviors, including: (a) a chaotic sea at low energy; (b) KAM islands; and (c) invariant spanning curves. See, for example, Refs. 23 and 24.

We will concentrate on the scaling behavior present in the chaotic sea at low energy. We use an extension of the formalism derived from surface science ${ }^{34}$ to investigate the behavior of the variance of the average energy which we will then refer to as the roughness, $\omega$. Application of this approach to the analysis of the time evolution of chaotic time-series ${ }^{35}$ has already led to a number of interesting results. We thus discuss in this paper the results of the roughness as function of the control parameter $N_{c}$ (see the abovegiven definition) and the connections with the previous results in the literature for a sort of system. Therefore, the results obtained as function of the control parameter $\delta$ allow us to characterize a special transition in this system.

Figure 2 shows the time series for a particular initial condition in the chaotic sea. It is immediately apparent that the time series looks rather similar, regardless of how the abscissa is expanded, suggesting scaling behavior. Figure 2 was constructed using the parameters $\delta=0.5, r=1$, and $N_{c}$ $=33.18$. Before formally defining the roughness, we first discuss the control parameters. The phase space must depend on $\delta, N_{c}$, and $r$ because the map $T$ is explicitly dependent on all three parameters. However, we will consider in this paper the case of a symmetrical potential, i.e., the case in which $a=b$, equivalent to taking $r=1$. Results for the case $r \neq 1$ will be published elsewhere.

In order to study the roughness, we first evaluate the energy (in the chaotic sea at low energy domain) averaged over the orbit generated from one initial condition as 


$$
\bar{e}\left(n, \delta, N_{c}\right)=\frac{1}{n} \sum_{i=1}^{n} e_{i}
$$

and then evaluate the interface width around this averaged energy. The roughness is then defined as

$$
\omega\left(n, \delta, N_{c}\right) \equiv \frac{1}{M} \sum_{j=1}^{M} \sqrt{\overline{e^{2}}{ }_{j}\left(n, \delta, N_{c}\right)-\bar{e}_{j}^{2}\left(n, \delta, N_{c}\right)},
$$

where we have considered an ensemble of $M$ different initial conditions on the chaotic sea. The scaling for the roughness will be discussed in Sec. III.

\section{SCALING PROPERTIES}

We will discuss the results obtained for the scaling in the chaotic sea as functions of each of the parameters $\delta$ and $N_{c}$. We start by considering the dependence on $N_{c}$.

\section{A. Scaling as function of $N_{c}$}

A scaling analysis for the roughness as a function of $N_{c}$ has been completed. It was carried out in a very similar way to that already presented in Ref. 5 so we discuss only the main points here, rather than providing the detailed arguments and demonstrating all the intermediate steps. As in Ref. 5, the roughness can be described formally in terms of a scaling function given by

$$
\omega\left(n, N_{c}\right)=l \omega\left(l^{a_{1}} n, l^{b_{1}} N_{c}\right),
$$

where $l$ is the scaling factor and $a_{1}$ and $b_{1}$ are the scaling dimensions. The exponents $a_{1}$ and $b_{1}$ should be related to the characteristic exponents $\alpha_{1}$ (roughening exponent), $\beta_{1}$ (growth exponent), and $z_{1}$ (dynamical exponent). Indeed, their relations are $-1 / a_{1}=\beta_{1},-1 / b_{1}=\alpha_{1}$. We also obtain that the critical exponents are related by

$$
z_{1}=\frac{a_{1}}{b_{1}}=\frac{\alpha_{1}}{\beta_{1}} .
$$

Following the procedures already described, ${ }^{5}$ we obtain $\alpha_{1}=0.667(2), \beta_{1}=0.50(1)$ (averaged over many roughness curves in a large window of $N_{c}$ ), and $z_{1}=1.318(9)$. We can also compare the numerical result obtained for $z_{1}$ with the result from Eq. (8). Evaluating Eq. (8) using both the previous results for the exponents $\alpha_{1}$ and $\beta_{1}$, we obtain $z_{1}$ $=1.33(2)$, which compares well with the above-presented numerical result.

As a final check on the validity of the scaling function and critical exponents, we collapse different roughness curves onto a single and universal roughness plot, as shown in Fig. 3 for different control parameters.

Before moving to the next section, we discuss the applicability of these exponents $\left(\alpha_{1}, \beta_{1}, z_{1}\right)$ to other systems. It is interesting to note that the results discussed here and those presented in Refs. 5 and 6 were all, in a sense, obtained as functions of the oscillation frequency. An increase of the frequency is quite equivalent to an increase in the "stochasticity" of these systems. Consequently, many of their observables require far more iteration to approach asymptotic values as compared to results obtained for lower oscillation
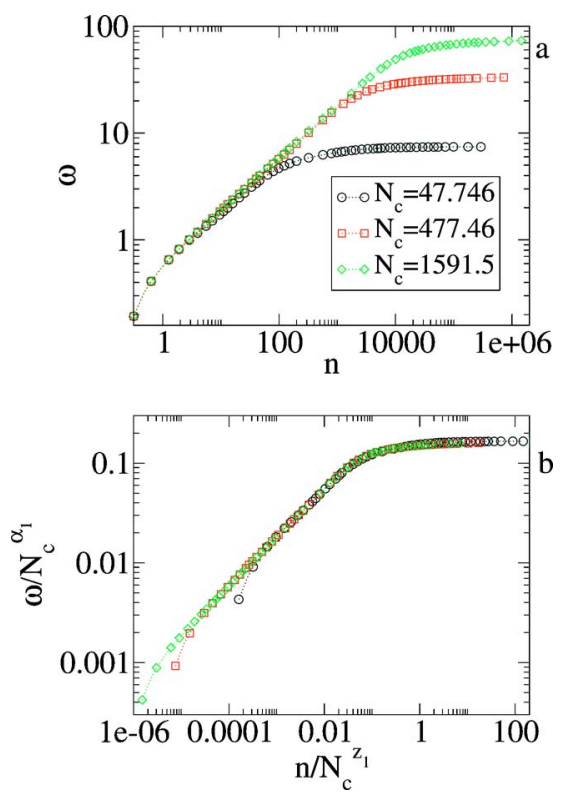

FIG. 3. (a) Roughness $\omega$ as a function of $n$ for different values of $N_{c}$. (b) Their collapse onto both the same saturation value and same crossover iteration number. For details, see Ref. 5.

frequencies. The rise in the lowest energy invariant spanning curve also implies an increase of the roughness saturation value. We can however observe that the roughness exponent $\beta_{1}$ seems to be independent of frequency and assumes the same value in different systems (cf. results for Refs. 5 and 6). In the case of the Fermi accelerator model, we have already demonstrated the existence of a situation ${ }^{35}$ in which the exponent $\beta$ does indeed depend on the initial velocity. We can even conclude that, within a range of uncertainty, the exponents $\alpha_{1}$ and $z_{1}$ obtained in this section are identical to those obtained in Refs. 5 and 6. The implication is that such exponents may be valuable in that they can be used to classify systems as falling within the same universality class. Other observables also contribute to and further support this idea, as follows. One can note that, in the problem of a timemodulated barrier, ${ }^{21,22}$ the traversal time, i.e. the length of time taken by the particle to traverse the oscillating barrier, obeys a power law distribution of exponent -3 . The same exponent has also been obtained for the distribution of successive reflection numbers and times for different periodic systems, including an oscillating single square well, ${ }^{23}$ a timemodulated barrier, ${ }^{5}$ and a double-square well potential. ${ }^{6}$ The authors of Ref. 23 also introduced an analytic approach to justify the exponent -3 for the case of a single square well moving randomly. These results, together with those obtained for the roughness and in relation to the exponents $\alpha_{1}$ and $z_{1}$, lend support to our hypothesis that such systems (i.e., at least those discussed in Refs. 5, 6, and 21-23 and even Ref. 24 for the case of infinite oscillating square wells) all belong to the same class of universality.

In the next section, we investigate for the first time how the roughness varies as a function of the depth of the oscillating potential. 

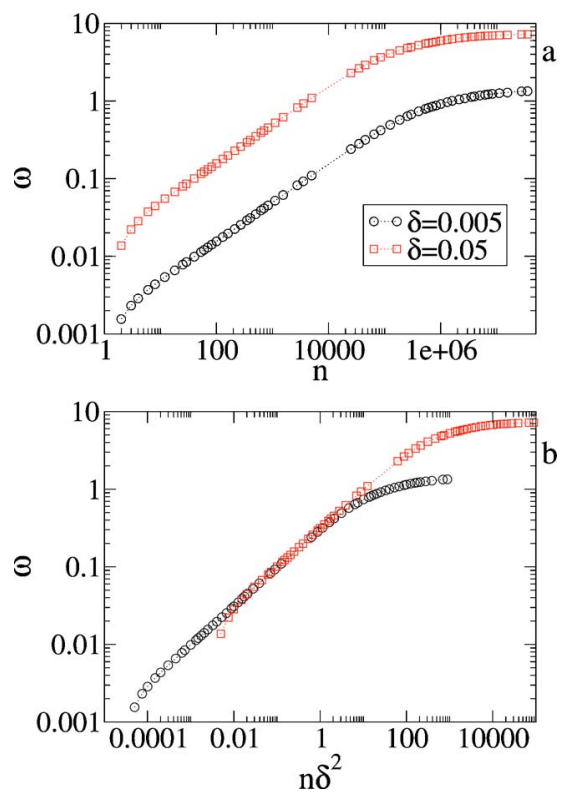

FIG. 4. Behavior of the roughness $\omega$ for an ensemble of $10^{4}$ initial conditions. The control parameters used were $N_{c}=795.77$ and two different values of $\delta$, namely $\delta=0.005$ and $\delta=0.05$. In (a), the roughness is plotted against the iteration number $n$ while (b) plots the roughness as a function of $n \delta^{2}$.

\section{B. Scaling as function of $\delta$}

We now consider the behavior of the roughness as a function of the control parameter $\delta$. Evaluation of Eq. (6) for different values of $\delta$ is shown in Fig. 4, where we have taken a fixed value of the parameter $N_{c}=795.77$. Note however that different values of $\delta$ generates different roughness curves for small $n$, as can be seen in Fig. 4(a). Such behavior indicates that $n$ is not a scaling variable. However, we can apply the transformation $n \rightarrow n \delta^{2}$ and thus observe that it coalesces both the curves for small $n$. This procedure is illustrated in Fig. 4(b). Figure 4 was obtained by averaging $10^{4}$ different initial phases and the same initial energy $e_{0}=1.001$, all of them leading to chaotic behavior.

As discussed in Ref. 5, it is easy to see in Fig. 4 that the roughness grows for small $n$, and then tends toward the saturation value as the iteration number becomes large enough. The change in growth rate marking the approach to saturation is characterized by a crossover iteration number. This discussion allows us to characterize the roughness as a function of the control parameter $\delta$, in a similar way to that used for $N_{c}$ in Sec. III A. We therefore suppose the following.

(1) After a brief initial transient and considering short iterations, the roughness is described as

$$
\omega\left(n \delta^{2}, \delta\right) \propto\left[n \delta^{2}\right]^{\beta_{2}},
$$

where $\beta_{2}$ is the growth exponent. Equation (9) is valid for $n \ll n_{x}$.

(2) Considering large enough iteration number, the saturation of roughness is given by

$$
\omega_{\text {sat }}\left(n \delta^{2}, \delta\right) \propto \delta^{\alpha_{2}},
$$

where $\alpha_{2}$ is the roughening exponent and Eq. (10) is valid for $n \gg n_{x}$.
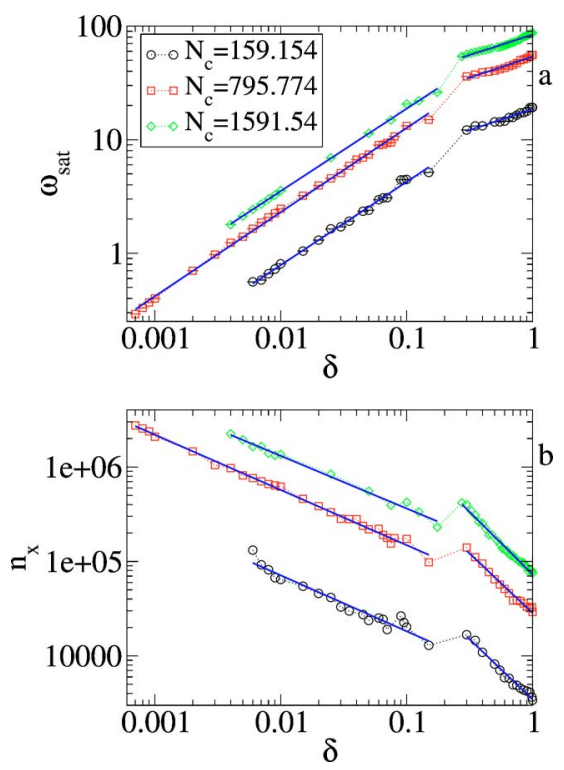

FIG. 5. (a) Plot of the saturation roughness $\omega_{\text {sat }}$ against the control parameter $\delta$. (b) The crossover iteration number as a function of $\delta$. In each case, we consider three different fixed values of the control parameter $N_{c}$, as indicated in (a).

(3) The crossover iteration number $n_{x}$ characterizing the change of growing and marking the approach to the saturation regime is given by

$$
n_{x}(\delta) \propto \delta^{2},
$$

where $z_{2}$ is the dynamical exponent.

In light of these three initial suppositions, the scaling function describing the roughness can be written as

$$
\omega\left(n \delta^{2}, \delta\right)=l \omega\left(l^{a_{2}} n \delta^{2}, l^{b_{2}} \delta\right),
$$

where $a_{2}$ and $b_{2}$ are the scaling dimensions and $l$ is the scaling factor. As discussed earlier, the exponents $a_{2}$ and $b_{2}$ can be related to the characteristic exponents $\alpha_{2}, \beta_{2}$, and $z_{2}$. To do so, we first write the scaling factor as $l=\left[n \delta^{2}\right]^{\left(-1 / a_{2}\right)}$. With this choice, we can rewrite Eq. (12) as

$$
\omega\left(n \delta^{2}, \delta\right)=\left[n \delta^{2}\right]^{\left(-1 / a_{2}\right)} \omega_{1}\left(\left[n \delta^{2}\right]^{\left(b_{2} / a_{2}\right)} \delta\right) .
$$

We define the function $\omega_{1}=\omega\left(1,\left[n \delta^{2}\right]^{\left(b_{2} / a_{2}\right)} \delta\right)$ and suppose it to be constant for $n \ll n_{x}$. Comparing Eqs. (9) and (13) we find that $-1 / a_{2}=\beta_{2}$. We now choose the scaling factor to be $l=\delta^{\left(-1 / b_{2}\right)}$. This allows us to rewrite Eq. (12) as

$$
\omega\left(n \delta^{2}, \delta\right)=\delta^{\left(-1 / b_{2}\right)} \omega_{2}\left(\delta^{\left(a_{2} / b_{2}\right)} n \delta^{2}\right) .
$$

The function $\omega_{2}=\omega\left(\delta^{\left(a_{2} / b_{2}\right)} n \delta^{2}, 1\right)$ and it is supposed constant for $n \gg n_{x}$. A direct comparison of Eqs. (10) and (14) shows that $-1 / b_{2}=\alpha_{2}$. To obtain the relationship between the critical exponents, we must consider the two different previous expressions for the scaling factor $l$. We can thus write

$$
n=\delta^{\left(a_{2} / b_{2}\right)-2} .
$$

Finally, comparing Eqs. (11) and (15), we obtain

$$
z_{2}=\frac{a_{2}}{b_{2}}-2=\frac{\alpha_{2}}{\beta_{2}}-2 .
$$

Figure 5 shows the behavior of the saturated roughness $\omega_{\text {sat }}$ 
TABLE I. Numerical exponents $\alpha_{2}$ and $z_{2}$ obtained for three different values of $N_{c}$.

\begin{tabular}{ccc}
\hline \hline$N_{c}$ & $\alpha_{2}$ & $z_{2}$ \\
\hline \multirow{3}{*}{159.154} & Before transition & \\
795.774 & $0.73(1)$ & $-0.59(3)$ \\
1591.54 & $0.741(6)$ & $-0.584(8)$ \\
& $0.726(8)$ & $-0.55(2)$ \\
& & \\
159.154 & After transition & $-1.27(5)$ \\
795.774 & $0.37(2)$ & $-1.26(6)$ \\
1591.54 & $0.37(2)$ & $-1.32(2)$ \\
\hline \hline
\end{tabular}

and the corresponding crossover iteration number $n_{x}$ as functions of the parameter $\delta$ for three different values of the control parameter $N_{c}$, as indicated in the figure. We can see however that the curves for both $\omega_{\text {sat }}$ and $n_{x}$ undergo a marked change in behavior near the value $\delta \approx 0.2$. For curves showing such a transition, we can still obtain numerically sets of the exponents $\alpha_{2}, \beta_{2}$, and $z_{2}$ separately for each of the two distinct regions. It is easy to see in Fig. 5 that the slope for each curve is quite different before and after the transition. The corresponding critical exponents $\alpha_{2}$ and $z_{2}$ are shown in Table I. After averaging over all the time series, we find that the exponent $\beta_{2}=0.498(3)$. Note that this result allows us to conclude that both the growth exponents are the same, i.e., $\beta_{1}=\beta_{2}=1 / 2$ for those sets of initial conditions discussed in the beginning of this section. It also assumes the same value in Refs. 5 and 6 and for the case where the initial velocities are very low in the Fermi accelerator model. ${ }^{35}$

We now compare the numerical results obtained for the critical exponents with the analytic relation Eq. (16). Before doing so, we first compute the average values of critical exponents obtained for the three different values of $N_{c}$ before and after the transition. Considering the roughness exponent before the transition, we find that its average value is $\bar{\alpha}_{2}^{b}$ $=0.732(8)$ and the dynamical exponent is $\bar{z}_{2}^{b}=-0.57(2)$. After the transition, the exponents are given by $\bar{\alpha}_{2}^{a}=0.366(2)$ and $\bar{z}_{2}^{a}=-1.28(4)$.

Evaluating Eq. (16), and taking into account the average values for $\alpha_{2}$ and $\beta_{2}$, we find: (i) that before the transition $z_{2}=-0.531(7)$ and (ii) that after the transition $z_{2}=-1.265(1)$. Our results show relatively good agreement for the exponents obtained both before and after the transition.

As a final check on the validity of this discussion, we try to collapse the roughness curves for different control parameters, considering the two different values obtained for the corresponding exponents $\alpha_{2}$ and $z_{2}$, as shown in Fig. 6. Figures 6(a) and 6(b) show results for the dynamics before the transition, while Figs. 6(c) and 6(d) shows the results after the transition. The collapses obtained in these figures confirm that the critical exponents give good results both before and after the transition.

Let us now discuss the transition observed near to $\delta$ $\approx 0.2$. It was shown earlier ${ }^{23}$ that the spectrum of Lyapunov exponents undergoes an abrupt transition as a function of the control parameters (see also Ref. 5 for the comparably abrupt transition of the Lyapunov exponent for a classical particle
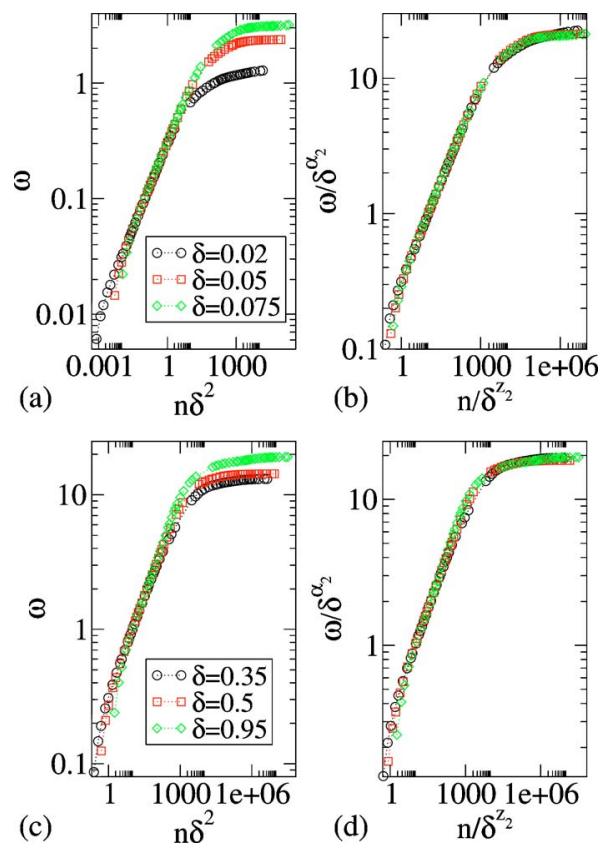

FIG. 6. Behavior of the roughness $\omega$ for different values of $\delta$ with the same control parameter $N_{c}=159.154$. (a) and (b) The results before the transition, (c) and (d) those obtained after the transition.

interacting with a time-modulated barrier). This abrupt transition was explained as rising from the destruction of the lowest invariant spanning curve and consequently the merging of two large but different chaotic regions. As we shall see, it is this transition that is responsible for the change in the behavior of the critical exponents. Figure 7(a), obtained for the control parameters $\delta=0.165$ and $N_{c}=159.154$, shows the two different large chaotic regions separated by an invariant spanning curve. After a tiny change of the parameter to $\delta=0.168$, Fig. 7(b) clearly possesses just a single large chaotic region.
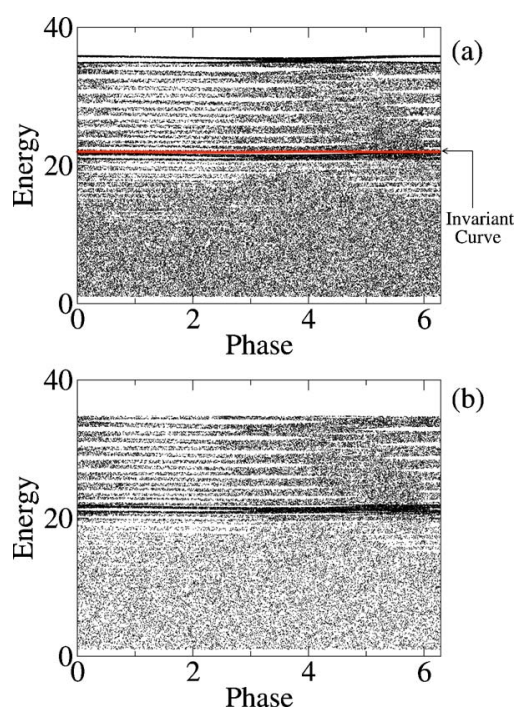

FIG. 7. (a) Iteration of three different initial conditions. The different chaotic regions are separated by an invariant spanning curve. The control parameters were $\delta=0.165$ and $N_{c}=159.154$. (b) Iteration from one initial condition for the control parameters $\delta=0.168$ and $N_{c}=159.154$. 

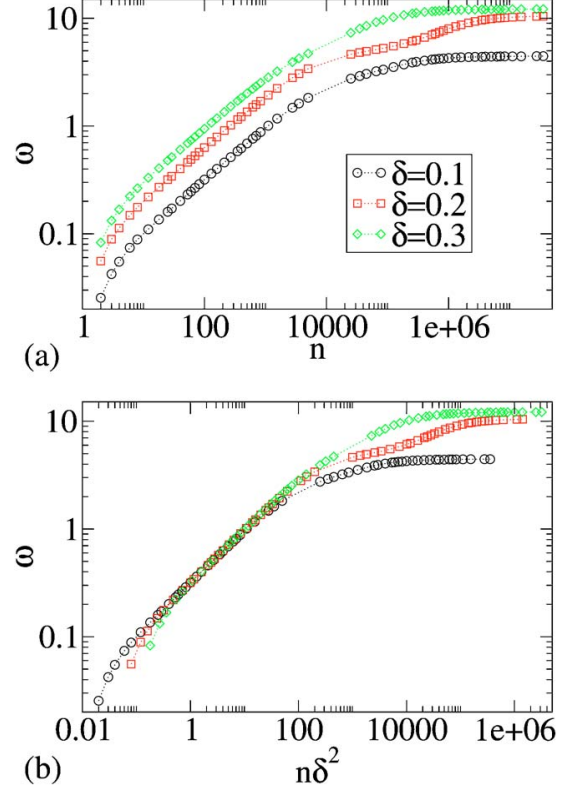

FIG. 8. Roughness evolution for three different values of $\delta$ and the same $N_{c}=159.154$ (a) as a function of $n$; and (b) as a function of $n \delta^{2}$.

The destruction of this invariant spanning curve has an immediate effect on the roughness curve, and consequently on the critical exponents. With the merging of the two different chaotic regions, the particles experience a transient in the previously chaotic region, behavior that affects directly the shape of the roughness curve, as can be seen in Fig. 8 for the control parameter $\delta=0.2$. It is easy to see however that, immediately after the transition, where the transient effects are stronger, the crossover iteration number is no longer easy to obtain and, as the roughness curve presents an extra bend, the collapse for different control parameters cannot occur. Figure 8 was obtained using three different values of $\delta$ (as illustrated in the figure) and the same $N_{c}=159.154$. Figure 8 (a) shows the three different roughness curves while Fig. 8(b) shows the same three curves but, after a brief initial transient and applying the transformation $n \rightarrow n \delta^{2}$, that all the curves coalesce together for short iterations. We emphasize that, even where the system undergoes sudden destruction of invariant spanning curves through the merging of at least two large chaotic regions, resulting in critical exponents that are different before and after the transition and when transient effects are not observed, the scaling laws given by Eqs. (7) and (12) still seem to be valid.

\section{SUMMARY AND CONCLUSIONS}

We have studied the problem of a classical particle interacting with an infinitely deep potential well containing a symmetric oscillating square well. The dynamics of the problem was described via a two-dimensional nonlinear areapreserving map. The chaotic low energy region was characterized via the formalism of a scaling function for the variance of the average energy (roughness). We have derived analytic relations for the critical exponents and shown that the expressions depend on which control parameter is being considered. The connections of the results presented in this paper with those previously reported in the literature have also been considered (see the end of Sec. III A) and their wider implications discussed.

\section{ACKNOWLEDGMENTS}

This research was supported by a grant from Conselho Nacional de Desenvolvimento Científico e Tecnológico$\mathrm{CNPq}$, Brazilian agency. The numerical results were obtained in the Centre for High Performance Computing in Lancaster University. The work was supported in part by the Engineering and Physical Sciences Research Council (UK).

${ }^{1}$ A. J. Lichtenberg and M. A. Lieberman, Regular and Chaotic Dynamics, Applied Mathematical Science Vol. 38 (Springer, New York, 1992).

${ }^{2}$ M. Tabor, Chaos and Intebrability in Nonlinear Dynamics-An introduction (Wiley, New York, 1989).

${ }^{3}$ M. V. Berry, Eur. J. Phys. 2, 91 (1981).

${ }^{4}$ E. D. Leonel, J. K. L. da Silva, and S. O. Kamphorst, Physica A 331, 435 (2004).

${ }^{5}$ E. D. Leonel and P. V. E. McClintock, Phys. Rev. E 70, 016214 (2004).

${ }^{6}$ E. D. Leonel and P. V. E. McClintock, J. Phys. A 37, 8949 (2004).

${ }^{7}$ G. A. Luna-Acosta, A. A. Krokhin, M. A. Rodríguez, and P. H. Hernández-Tejeda, Phys. Rev. B 54, 11410 (1996).

${ }^{8}$ G. A. Luna-Acosta, K. Na, L. E. Reichl, and A. Krokhin, Phys. Rev. E 53, 3271 (1996).

${ }^{9}$ G. A. Luna-Acosta, J. A. Méndez-Bermúdez, P. Seba, and K. N. Pichugin, Phys. Rev. E 65, 046605 (2002).

${ }^{10}$ M. Robnik and M. V. Berry, J. Phys. A 18, 1361 (1985).

${ }^{11}$ N. Saitô, H. Hirooka, J. Ford, F. Vivaldi, and G. H. Walker, Physica D 5, 273 (1982).

${ }^{12}$ K. Y. Tsang and K. L. Ngai, Phys. Rev. E 56, R17 (1997).

${ }^{13}$ R. E. de Carvalho, Phys. Rev. E 55, 3781 (1997).

${ }^{14}$ A. Loskutov, A. B. Ryabov, and L. G. Akinshin, J. Phys. A 33, 7973 (2000).

${ }^{15}$ M. Buttiker and R. Landauer, Phys. Rev. Lett. 49, 1739 (1982).

${ }^{16}$ M. Wagner, Phys. Rev. B 57, 11899 (1998).

${ }^{17}$ L. P. Kouwenhoven, S. Jauhar, J. Orenstein, P. L. McEuen, Y. Nagamune, J. Motohisa, and H. Sakaki, Phys. Rev. Lett. 73, 3443 (1994).

${ }^{18}$ P. S. S. Guimarães, B. J. Keay, J. P. Kaminski, S. J. Allen Jr., P. F. Hopkins, A. C. Gossard, L. T. Florez, and J. P. Harbison, Phys. Rev. Lett. 70, 3792 (1993)

${ }^{19}$ W. Cai, P. Hu, T. F. Zheng, B. Yudanin, and M. Lax, Phys. Rev. B 41, 3513 (1990)

${ }^{20}$ A. O. Caldeira and A. J. Leggett, Phys. Rev. Lett. 46, 211 (1981).

${ }^{21}$ J. L. Mateos and J. V. José, Physica A 257, 434 (1998).

${ }^{22}$ J. L. Mateos, Phys. Lett. A 256, 113 (1999).

${ }^{23}$ E. D. Leonel and J. K. L. da Silva, Physica A 323, 181 (2003).

${ }^{24}$ G. A. Luna-Acosta, G. Orellana-Rivadeneyra, A. Mendoza-Galván, and C. Jung, Chaos, Solitons Fractals 12, 349-363 (2001).

${ }^{25}$ V. Berdichevsky and M. Gitterman, Phys. Rev. E 53, 1250 (1996).

${ }^{26}$ V. Berdichevsky and M. Gitterman, J. Phys. A 29, 1567 (1996).

${ }^{27}$ V. Berdichevsky and M. Gitterman, Phys. Rev. E 59, R9 (1999).

${ }^{28}$ M. I. Dykman, B. Golding, L. I. McCann, V. N. Smelyanskiy, D. G. Luchinsky, R. Mannella, and P. V. E. McClintock, Chaos 11, 587 (2001).

${ }^{29}$ V. Berdichevsky and M. Gitterman, J. Phys. A 29, L447 (1996).

${ }^{30}$ M. Arrayás, I. Kh. Kaufman, D. G. Luchinsky, P. V. E. McClintock, and S. M. Soskin, Phys. Rev. Lett. 84, 2556 (2000).

${ }^{31}$ J. Iwaniszewski, Phys. Rev. E 68, 027105-1 (2003).

${ }^{32}$ J. Iwaniszewski, I. Kh. Kaufman, P. V. E. McClintock, and A. J. McKane, Phys. Rev. E 61, 1170 (2000).

${ }^{33}$ J. Iwaniszewski, Phys. Rev. E 54, 3173 (1996).

${ }^{34}$ A.-L. Barabási and H. E. Stanley, Fractal Concepts in Surface Growth (Cambridge University Press, Cambridge, 1995).

${ }^{35}$ E. D. Leonel, P. V. E. McClintock, and J. K. L. da Silva, Phys. Rev. Lett. 93, 014101 (2004).

${ }^{36}$ W. Cai, T. F. Zheng, P. Hu, B. Yudanin, and M. Lax, Phys. Rev. Lett. 63, 418 (1989). 